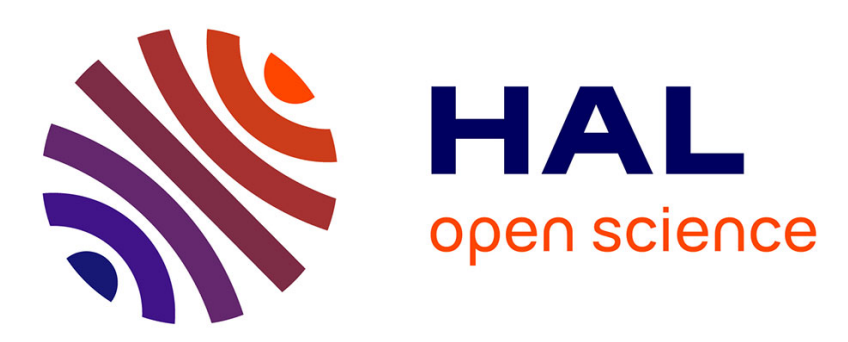

\title{
Extraction of state-resolved information from systems with a fractional number of electrons within the framework of time-dependent density functional theory.
}

Feng Wang, Yugui Yao, Florent Calvayrac, Feng Zhang

\section{- To cite this version:}

Feng Wang, Yugui Yao, Florent Calvayrac, Feng Zhang. Extraction of state-resolved information from systems with a fractional number of electrons within the framework of time-dependent density functional theory.. Journal of Chemical Physics, 2016, 145 (11), pp.114104. 10.1063/1.4962908 . hal-01906078

HAL Id: hal-01906078

https://hal-univ-lemans.archives-ouvertes.fr/hal-01906078

Submitted on 17 Dec 2018

HAL is a multi-disciplinary open access archive for the deposit and dissemination of scientific research documents, whether they are published or not. The documents may come from teaching and research institutions in France or abroad, or from public or private research centers.
L'archive ouverte pluridisciplinaire HAL, est destinée au dépôt et à la diffusion de documents scientifiques de niveau recherche, publiés ou non, émanant des établissements d'enseignement et de recherche français ou étrangers, des laboratoires publics ou privés. 
Extraction of state-resolved information from systems with a fractional number of electrons within the framework of time-dependent density functional theory

Feng Wang', Yugui Yao, Florent Calvayrac, and Fengshou Zhang

Citation: The Journal of Chemical Physics 145, 114104 (2016); doi: 10.1063/1.4962908

View online: http://dx.doi.org/10.1063/1.4962908

View Table of Contents: http://aip.scitation.org/toc/jcp/145/11

Published by the American Institute of Physics

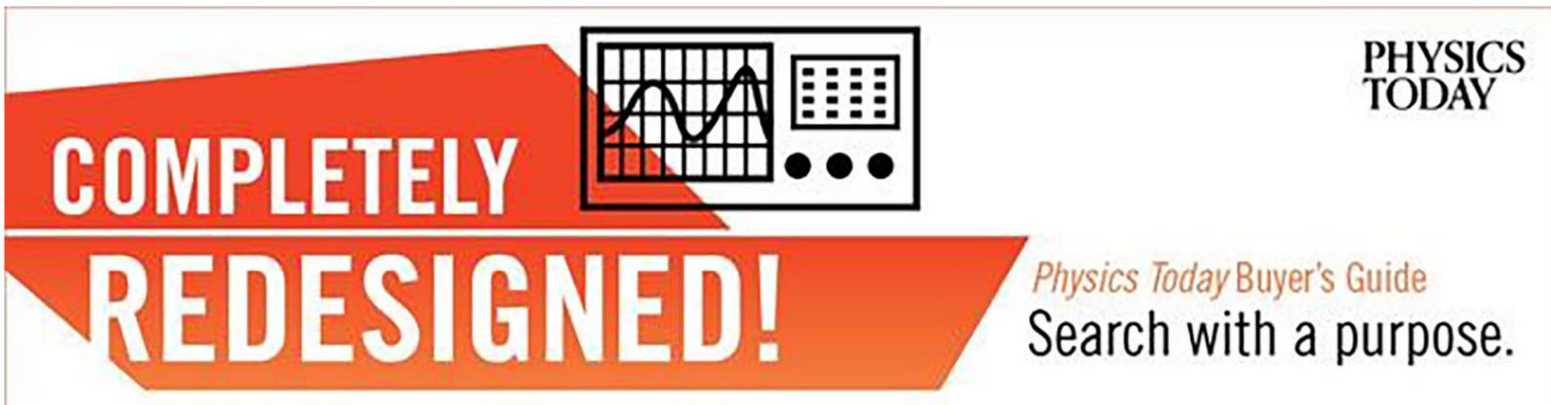




\title{
Extraction of state-resolved information from systems with a fractional number of electrons within the framework of time-dependent density functional theory
}

\author{
Feng Wang, ${ }^{1, a)}$ Yugui Yao, ${ }^{1}$ Florent Calvayrac, ${ }^{2}$ and Fengshou Zhang ${ }^{3}$ \\ ${ }^{1}$ School of Physics, Beijing Institute of Technology, Beijing 100081, China \\ ${ }^{2}$ Institut des Molecules et Matériaux du Mans UMR 6283, Université du Maine, LUNAM 72085 Le Mans \\ Cedex 9, France \\ ${ }^{3}$ The Key Laboratory of Beam Technology and Material Modification of Ministry of Education, College \\ of Nuclear Science and Technology, Beijing Normal University, Beijing 100875, China
}

(Received 17 July 2016; accepted 5 September 2016; published online 20 September 2016)

\begin{abstract}
The determination of the state-resolved physical information within the framework of time-dependent density functional theory has remained a widely open question. We demonstrated the ability to extract the state-resolved probability from the knowledge of only the time-dependent density, which has been used as the basic variable within the time-dependent density functional theory, with the help of state-resolved single-electron capture experiments for collisions of protons on helium in the energy range of 2-100 keV/amu. The present theoretical results for capture into states of $\mathrm{H}(1 s), \mathrm{H}(2 s)$, and $\mathrm{H}(2 p)$ are in good agreement with the most sophisticated experimental results of $\mathrm{H}^{+}+\mathrm{He}\left(1 s^{2}\right)$ system, validating our approach and numerical implementation. Published by AIP Publishing. [http://dx.doi.org/10.1063/1.4962908]
\end{abstract}

\section{INTRODUCTION}

By virtue of its computational advantages, timedependent density functional theory (TDDFT) ${ }^{1-4}$ is among the most powerful quantum mechanical approaches to access on a first-principles basis, the regime of the nonlinear and the nonperturbative response in a strong-excitation process, where TDDFT in principle has huge advantages and is most urgently needed. In practice, however, applications of TDDFT are hampered by the lack of exact knowledge about two essential ingredients, namely the time-dependent exchange-correlation (XC) potential and the functional for the physical observable. On the one hand, most implementations of TDDFT utilize the adiabatic approximation where, groundstate density functionals are used in the calculation of the time-dependent XC potential. Accordingly, the failures of the time-dependent XC potential were all traced back to its lack of nonlocality in space and time. On the other hand, even when the time-dependent XC potential (and, therefore, the one-body electron density) was known exactly, the calculation of physical observables would still be a non-trivial problem. Within the framework of TDDFT, the one-body electron density replaces the many-body wave-function as the basic variable. The Runge-Gross theorem ${ }^{5}$ assures that the one-body electron density determines all observables, which means that all physical quantities of interest can in principle be expressed exactly as a functional of the one-body electron density. In fact, it turns out that some observables may be obtained quite straightforwardly in that fashion, whereas others are rather cumbersome to express as explicit functionals of the one-body electron density, for which a suitable algorithm needs to be found.

\footnotetext{
a)wangfeng01@ $@$ tsinghua.org.cn
}

A prominent example is a theoretical description for a strong-excitation process of a finite system. When a finite system is exposed to strong excitations such as irradiation with high-intensity laser pulses or collisions with fast, highly charged ionic projectiles, some of the electronic density can move far away from the systems center. Simulating this process in TDDFT typically leads to a situation where the density that remains bound integrates to a fractional number of electrons, ${ }^{6,7}$ and no accurate functionals for the state-resolved probabilities of systems are known. Fortunately, the existence of abundant experimental data for atomic collision systems could in turn help to guide theoretical development. Thus, extracting the state-resolved physical information directly from the one-body electron density is an important subject from both fundamental and technological points of view. At present, there are already some efforts underway ${ }^{8-11}$ along this way.

In this work, we therefore focus our attention on the open problem of designing algorithms for the extraction of the state-resolved probabilities from the time-dependent one-body electron density. We formulate a novel conceptual development that allows the determination of the stateresolved probabilities of the ionization and excitation of systems in a uniform fashion, and depends only on the time-dependent one-body electron density in the spirit of TDDFT. After that, we have tested this new method with the help of state-resolved single-electron capture experiments for collisions of protons on helium in the energy range of 2-100 keV/amu.

\section{METHOD}

We shall now introduce our algorithms for the extraction of the state-resolved probabilities from the time-dependent 
one-body electron density (atomic units with $m=|e|=\hbar=1$ are used throughout this work unless otherwise indicated). Let us consider a time-dependent $N$-electron system under the influence of an external perturbation that is switched off at time $t$. The system then evolves freely in time $t^{\prime}$, where $t^{\prime}>t$. The starting point is the expansion of the exact timedependent state-vector $\left|\Psi_{t}\left(t^{\prime}\right)\right\rangle$ of the system in terms of the complete set $\left\{\left|\Phi_{m}\right\rangle, m=1,2, \ldots\right\}$ of stationary many-body wave functions of the system in absence of the external perturbation as

$$
\left|\Psi_{t}\left(t^{\prime}\right)\right\rangle=\sum_{m} C_{m}^{N}(t)\left|\Phi_{m}\right\rangle e^{-i \omega_{m} t^{\prime}},
$$

where $C_{m}^{N}(t)$ and $\omega_{m}$ are the expansion coefficients, and the corresponding eigen-frequencies of these eigen-states. The density $\rho_{t}\left(\mathbf{r}, t^{\prime}\right)$, which involves the information compression from the many-body density to the one-body density, is defined as

$$
\begin{aligned}
\rho_{t}\left(\mathbf{r}, t^{\prime}\right) & =\left\langle\Psi\left|\sum_{\mathbf{i}=1}^{N} \delta\left(\hat{\mathbf{r}}_{i}-\hat{\mathbf{r}}\right)\right| \Psi\right\rangle \\
& =\sum_{n} D_{n, n}(\mathbf{r}, t)+\sum_{m} \sum_{n, n \neq m} D_{m, n}(\mathbf{r}, t) e^{i\left(\omega_{m}-\omega_{n}\right) t^{\prime}}
\end{aligned}
$$

where the notation $\hat{\mathbf{r}}_{i}$ denotes the operator of space coordinate $\mathbf{r}$ for the $i$ th electron, and

$$
\begin{aligned}
D_{m, n}(\mathbf{r}, t) & =C_{m}^{N *}(t) C_{n}^{N}(t) \rho_{m, n}^{N}(\mathbf{r}) \\
& =C_{m}^{N *}(t) C_{n}^{N}(t)\left\langle\Phi_{m}\left|\sum_{\mathbf{i}=1}^{N} \delta\left(\hat{\mathbf{r}}_{i}-\hat{\mathbf{r}}\right)\right| \Phi_{n}\right\rangle .
\end{aligned}
$$

Here $\rho_{m, n}^{N}(\mathbf{r})$ is the diagonal elements of the transition matrices $^{12}$ and $\rho_{m, m}^{N}(\mathbf{r})$ is the eigen-densities $\rho_{m}^{N}(\mathbf{r})$ of the $m$ th eigen-state of the $N$-electron system.

In the non-degenerate case of $\omega_{m} \neq \omega_{n}$ for $m \neq n$, the $t^{\prime}$ time-averaged density may be expanded in the form, ${ }^{9}$

$$
\lim _{\tau \rightarrow \infty} \frac{1}{\tau} \int_{0}^{\tau} \rho_{t}\left(\mathbf{r}_{i}, t^{\prime}\right) d t^{\prime}=\sum_{m} P_{m}^{N}(t) \rho_{m}^{N}\left(\mathbf{r}_{i}\right),
$$

at some sampling points $\left\{\mathbf{r}_{i}, i=1, \ldots, L\right\}$. Equation (4) provides a recipe to extract the occupation probability $P_{m}^{N}(t)=\left|C_{m}^{N}(t)\right|^{2}$ of the respective $N$-electron eigen-densities from $\rho_{t}\left(\mathbf{r}, t^{\prime}\right)$. Furthermore, the solution of Equation (4) can be transformed into a problem of the constrained fitting, i.e., we have fitted the right-hand side of these equations to their left hand side with $P_{m}^{N}(t)$ as adjustable parameters.

As of today, it is not possible to extract the stateresolved ionization probabilities directly from the density in an elementary way. After the strong excitations, in order to determine the ionization of the initial $N$-electron system, including the case of the ionization-states, each of which contains a different number of electrons $n$ where $n \leq N$, Equation (4) has been generalized, being partly inspired by the ensemble description of electronic structure problems of a system with a noninteger number of electrons, ${ }^{13}$ as

$$
\lim _{\tau \rightarrow \infty} \frac{1}{\tau} \int_{0}^{\tau} \rho_{t}\left(\mathbf{r}_{i}, t^{\prime}\right) d t^{\prime} \Longleftarrow \sum_{n=1}^{N} \sum_{m=1}^{M_{n}} P_{m}^{n}(t) \rho_{m}^{n}\left(\mathbf{r}_{i}\right),
$$

where the symbol $\Leftarrow$ stands for the phrase: "is fitted to," $M_{n}$ is the dimension of truncated state space, and $P_{m}^{n}(t)$ is the occupation probability of the $m$ th eigen-state of the $n$-electron system. We would also like to mention that Equation (5) can be rigorously derived starting from the many-body wave function, along similar lines as in Ref. 14. In fact, the power of the fitting procedure is out of the ordinary, yielding one key benefit: it enables Equation (4) can be generalized straightforwardly to ionization case. Moreover, as a matter of principle, one can always find a unitary transformation that makes the spatial patterns of the degenerate eigen-densities different from each other, yielding another key benefit: it enables Equation (4) also can be applied to degenerate case.

It is important to note that solving Equation (5) on a series of random samples of size $L$ of space coordinate $\mathbf{r}$, and consequently from that, statistically averaging, what is a more accurate and unbiased estimation of $P_{m}^{n}(t)$. One of the key findings of our work is that Equation (5) produces results within the uncertainty of the experiments as long as the number of sampling points, $L\left(N_{a}\right)$, was chosen appropriately as a function of average number of electrons remaining in bound states, $N_{a}$, within the finite volume $V$ centered around the ionic core, with the basic relation,

$$
N_{a}(t)=\int_{V} \rho_{t}(\mathbf{r}) d \mathbf{r}=\sum_{n=1}^{N} n \sum_{m=1}^{M_{n}} P_{m}^{n}(t) .
$$

The basic idea to introduce this factor is in order to avoid overfitting, ${ }^{15}$ as it can exaggerate minor errors in the data. For a specific $L\left(N_{a}\right)$ presented in this paper, we refer the reader to Equation (8). Here and in what follows, we would like to refer the algorithm presented here as density-fitting analysis (DFA), which is intuitively very plausible, and can be judged a posteriori by the applications.

\section{APPLICATIONS}

To validate the applicability of DFA algorithm presented above, the $\mathrm{H}^{+}+\mathrm{He}\left(1 s^{2}\right)$ colliding system has been chosen serving as a benchmark. In order to describe the microscopic collision processes, we employ Ehrenfest dynamics, ${ }^{16}$ in which the electron dynamics follows TDDFT, while the movement of the ionic cores follows the classical Newton's law. For an unbiased representation, the Kohn-Sham orbitals and densities are discretized on a uniform mesh inside a real-space rectangular box with three side-lengths of 60,60 , and 120 a.u. whose size is sufficiently large to support orbitals with the principal quantum number of 1 and 2 for hydrogen atom. ${ }^{17}$ The real-time propagation was performed with the enforced time reversal symmetry method. ${ }^{18}$ Since the timestep is controlled by the mesh-spacing, we use an optimized time-step of 0.01 a.u. for a selected mesh-spacing of 0.3 a.u. under a condition that the time propagation is unitary. The $y-z$ plane is chosen as the scattering plane, taking the $z$-direction as the incident direction. The system has been propagated 
from an initial separation between projectile and target cores of 70 a.u. to the same final distance in the $z$-direction, and 20 impact parameters between $b_{\min }=0.5 \mathrm{a}$.u. and $b_{\max }=6.5 \mathrm{a}$.u. in the $y$-direction have been considered for each impact energy.

After the collision, the wave function of the system becomes a wave packet, which is a coherent superposition of those states that will survive in the interaction. Then this wave packet expands gradually during its time evolution. Although a given lattice can provide a high-fidelity representation of the initial, relatively small, wave packet, it indeed cannot directly or completely represent arbitrary highly excited or continuum states whose spatial extents go beyond the boundaries of the numerical grid. Provided that the time propagation is carried out to a large scale, the fraction of the wave function associated with highly excited or continuum states can be removed by absorbing boundary condition ${ }^{19}$ that was placed at the boundary of the simulation box. The absorption leads to a loss of the number of electrons remaining in the simulation box, $N_{a}^{\mathrm{H}}(t)+N_{a}^{\mathrm{He}}(t)$, where $N_{a}^{\mathrm{H}}(t)$ and $N_{a}^{\mathrm{He}}(t)$ are average numbers of electrons around proton and $\mathrm{He}$ core, respectively, which have nonintegral values in general, so we have to interpret them in a probabilistic sense with the aid of Equation (6). The convergence of the calculations with respect to selected numerical parameters has been demonstrated in previous wide applications. $^{20-26}$

The $\mathrm{H}^{+}+\mathrm{He}\left(1 s^{2}\right)$ colliding system consists of 2 active electrons and 2 ionic cores. The interaction between ionic cores and active electrons is described by means of a norm-conserving pseudopotential ${ }^{27}$ generated by a software package APE (version 2.2.0). ${ }^{28}$ The pseudopotential removes the singularity at the nucleus, and thus can be expressed accurately on a relatively coarse real-space grid. We here simulate the collision process at different impact parameters using a software package OCTOPUS (version 4.1.2) ${ }^{29,30}$ with $\mathrm{XC}$ potential under the adiabatic local-density approximation $(\text { ALDA })^{31}$ parameterized by the Perdew-Zunger ${ }^{32}$ scheme. In order to apply DFA algorithm, the eigen-densities involved must be specified. Although the eigen-density of the excited state is generally not readily accessible from TDDFT, it can be supplied by the other electronic structure methods, such as quantum chemistry methods. Therefore, this does not represent one major limitation to the applicability of DFA algorithm. In addition, some desired eigen-densities can be approximately calculated from TDDFT, using a technique of Ref. 11 in the appropriate limit. For the present study, only the eigen-densities of $\mathrm{H}(1 s), \mathrm{H}(2 s)$, and $\mathrm{H}(2 p)$ states were needed, and because of its one-electron simplicity, were easily determined by the direct solution of the stationary Schrödinger equation using APE software package.

In the following, we briefly report the results of some calculations. To this end, we first demonstrate the collision of $\mathrm{H}^{+}+\mathrm{He}\left(1 s^{2}\right)$ at an incident energy of $15 \mathrm{keV} / \mathrm{amu}$ with an impact parameter of 2.5 a.u. Figure 1 gives first insight into the collision process showing the spatially and time-resolved electron density distribution in order from (a) to (h). During the collision, the electronic distribution extending both the helium core and the proton is seen from (c) to (d). While approaching the helium core, the proton accumulates some charge around it. It then scatters and leaves the helium core picking up a part of the electronic charge. In (h), which is the final stage of the collision, the proton is seen to possess a spatially extended electron cloud, suggesting the formation of an excited hydrogen configuration. Since the space of the simulation box is limited, after (b) two translational transforms in both momentum and coordinate ${ }^{21}$ are conducted to the electrons and ionic cores so that the proton always stays inside the simulation box. After the collision has concluded, the electron densities centered around the ionic cores show slow rotations and fast oscillations exhibiting beatings between finite number of survived eigen-states, as shown in Figure 2. Clearly, to cover more eigen-states will require even larger sizes of simulation box, i.e., in a practical application, the summation over eigen-states in Equation (5) will therefore be truncated according to the finite size of the simulation box in a straightforward manner. For the present study, the size of the simulation box is chosen appropriately to support the eigen-states of $\mathrm{H}(1 s), \mathrm{H}(2 s)$, and $\mathrm{H}(2 p)$.

It thus follows that the cross section of electron capture into the $\mathrm{H}(\chi)$ state for a given impact energy is given by

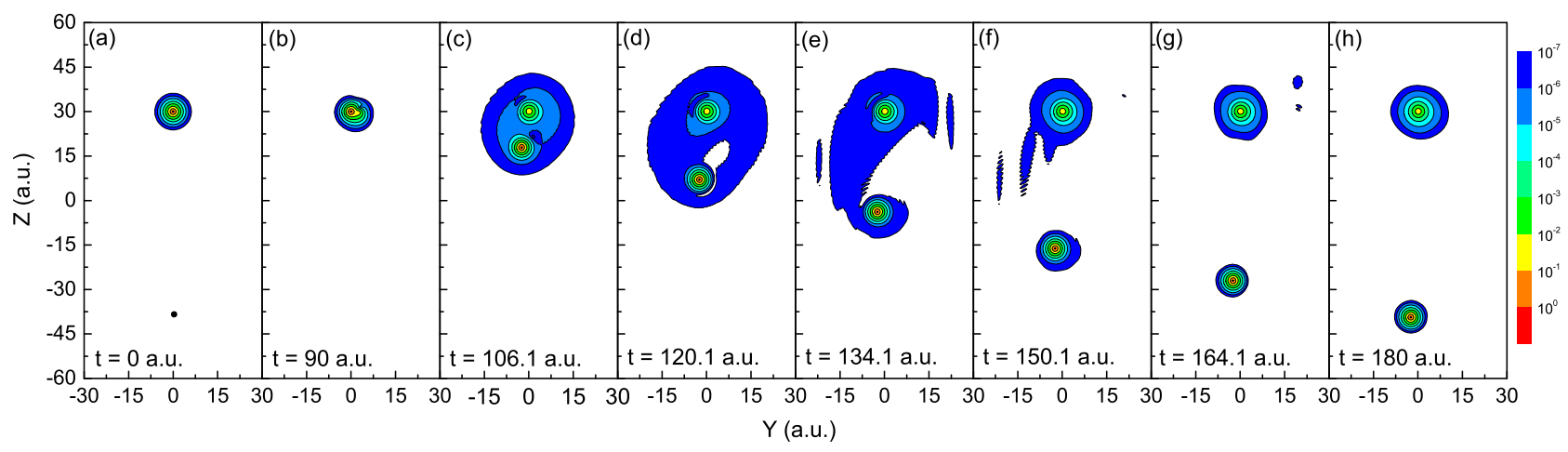

FIG. 1. Time development of the electronic density distribution in the scattering plane of $x=0$ a.u. in the collision of $\mathrm{H}^{+}+\mathrm{He}\left(1 s^{2}\right)$ at an incident ion energy of $15 \mathrm{keV} / \mathrm{amu}$ and impact parameter of 2.5 a.u. The projectile and target are separated by 70 a.u. in the incident $z$-direction in (a), closest approach in (b), and the final stage of the calculation (h) where the two ions are again separated by 70 a.u. in the incident $z$-direction. The initial projectile position is depicted as $\bullet$ in (a). The contour plot of the electronic density is scaled logarithmically. 


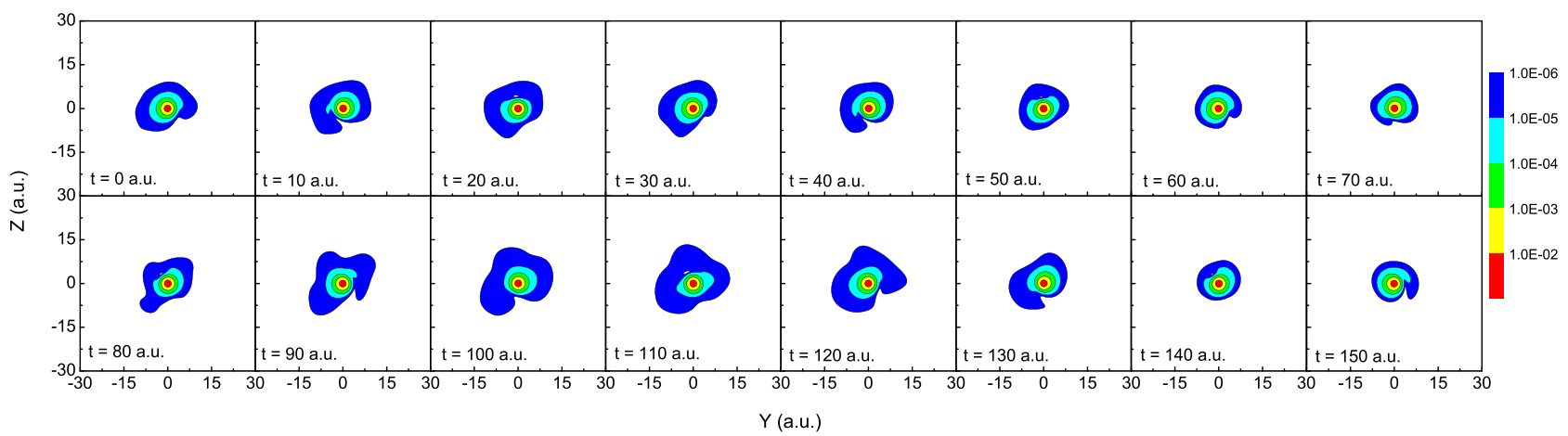

FIG. 2. The free time evolution of electron density distribution around the projectile at an incident ion energy of $15 \mathrm{keV} / \mathrm{amu}$ and impact parameter of 1.5 a.u. after the collision. The contour plot of the electronic density is scaled logarithmically.

$$
\sigma_{\chi}=2 \pi \int_{b_{\min }}^{b_{\max }} P_{\chi}^{1}(b) b d b
$$

where $P_{\mathcal{X}}^{1}(b)$ is the capture probability for a given projectile velocity and impact parameter $b$.

A quantity of central importance to DFA algorithm is the number of sampling points $L\left(N_{a}\right)$. In the present study, for capture into states of $\mathrm{H}(1 s), \mathrm{H}(2 s)$, and $\mathrm{H}(2 p)$ of $\mathrm{H}^{+}+\mathrm{He}\left(1 s^{2}\right)$ system, the following choice of $L\left(N_{a}^{\mathrm{H}}\right)$ seems to work best (and is determined once and for all):

$$
L\left(N_{a}^{\mathrm{H}}\right)=C_{0}+\sum_{n=1}^{2} \frac{C_{1, n}}{\exp \left[C_{2, n}\left(N_{a}^{\mathrm{H}}-C_{3, n}\right)\right]+1},
$$

with $C_{0}=30, C_{1,1}=300, C_{2,1}=25, C_{3,1}=0.1, C_{1,2}=300$, $C_{2,2}=30$, and $C_{3,2}=0.01$, respectively, by sampling only in regions of space where at least one of the eigen-densities more than $1.0 \times 10^{-5}$ is located.

The cross sections obtained with DFA algorithm for electron capture into the $\mathrm{H}(2 p)$ state are compared to the experimental results in Figure 3. We see that at impact energies below $10 \mathrm{keV} / \mathrm{amu}$, the other experimental data exhibit a less steep decrease with decreasing energy, compared to the results of Hippler et al. ${ }^{33}$ and Van Zyl et al., ${ }^{39}$ corrected

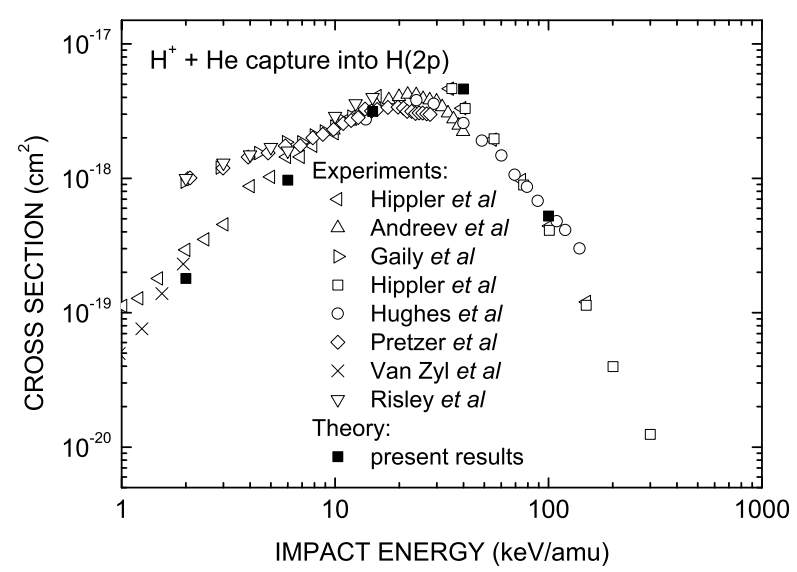

FIG. 3. Cross sections for electron capture into $\mathrm{H}(2 p)$ in $\mathrm{H}^{+}$on $\mathrm{He}\left(1 s^{2}\right)$. Experiment: $\triangleleft$, Hippler et al. $;{ }^{33} \Delta$, Andreev et al. $;^{34} \triangleright$, Gaily et al. $;^{35} \square$, Hippler et al. $;^{36} \circ$, Hughes et al. $;^{37} \diamond$, Pretzer et al. $;^{38} \times$, Van Zyl et al. ${ }^{39}$ $\nabla$, Risley et al. ${ }^{40}$ Theory: $\mathbf{m}$, present data. for experimental errors caused by charge changing collisions with the background gas. ${ }^{41}$ Note that in the energy range of approximately $2-200 \mathrm{keV} / \mathrm{amu}$ the uncertainty is about $20 \%$ for $\mathrm{H}(2 p)$, at lower and higher energies the experimental data are less certain. ${ }^{41}$

The cross sections obtained with DFA algorithm for electron capture into $\mathrm{H}(2 \mathrm{~s})$ and $\mathrm{H}(1 \mathrm{~s})$ states are compared to the experimental results in Figures 4 and 5, respectively. Since the charge transfer mainly populates the $\mathrm{H}(1 s)$ ground state in the $\mathrm{H}^{+}+\mathrm{He}\left(1 s^{2}\right)$ system, we compare the charge-transfer cross sections for the $\mathrm{H}(\mathrm{ls})$ state obtained from the present method to experimental total capture cross sections which include small contributions from excited states.

The present results of the cross sections, obtained with DFA algorithm, agree quite well with experimental data for incident energies above $\sim 5 \mathrm{keV} / \mathrm{amu}$ in the energy range of 2-100 keV/amu. This is a strong indication that the DFA algorithm captures the essential features which contribute to a sensible description of a phenomenon which crucially involves excited states of the system. For lower impact energies, the discrepancy between theory and experiment is relatively large. One possible reason may be the application of the pseudopotential. The pseudopotential is not expected to be valid for describing collisions

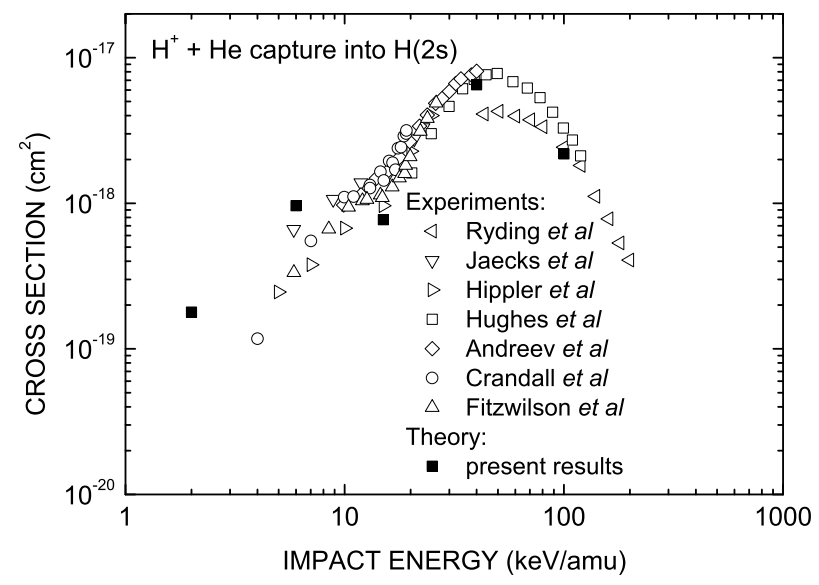

FIG. 4. Cross sections for electron capture into $\mathrm{H}(2 s)$ in $\mathrm{H}^{+}$on $\mathrm{He}\left(1 s^{2}\right)$. Experiment: $\triangleleft$, Ryding et al. ${ }^{42} \nabla$, Jaecks et al. ${ }^{43} \triangleright$, Hippler et al. $;^{44} \square$, Hughes et al. $;^{37} \diamond$, Andreev et al. ${ }^{34} \circ$, Crandall et al. ${ }^{45} \Delta$, Fitzwilson et al. ${ }^{46}$ Theory: $\mathbf{}$, present data. 


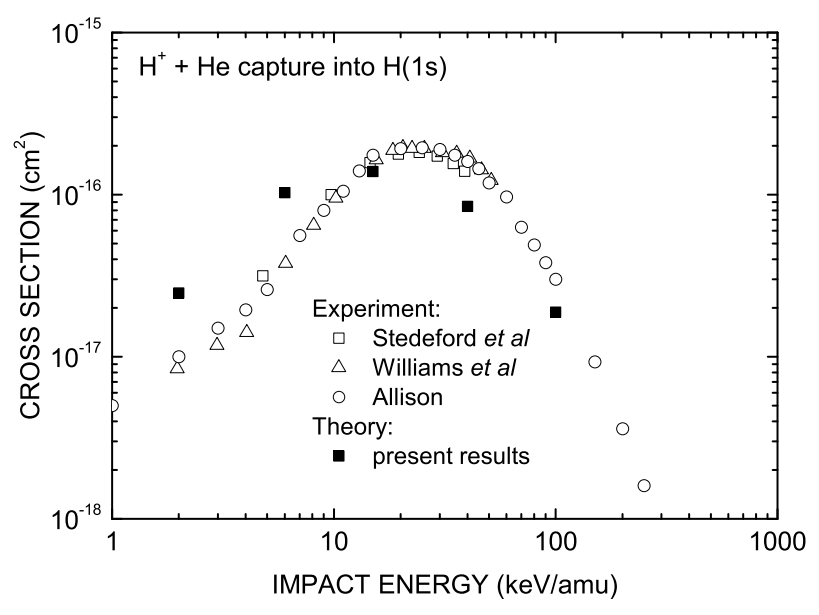

FIG. 5. Cross sections for electron capture into $\mathrm{H}(1 s)$ in $\mathrm{H}^{+}$on $\mathrm{He}\left(1 s^{2}\right)$. Experiment: $\square$, Stedeford et al. $;^{47} \Delta$, Williams et al. ${ }^{48}$ O, Allison. ${ }^{49}$ Theory: -, present data.

at small impact parameters where the overlap between the pseudopotential cores is non-negligible, and thus the calculated total cross section is inaccurate for lower impact energies because of the large contribution from small impact parameters. At higher energies, i.e., near the peak of these cross sections, the pseudopotential results are less inaccurate because of the large contribution from larger impact parameters.

In TDDFT, all physical observables of interest should be calculated from the time-dependent density, while the timedependent Kohn-Sham orbitals (KSO) are merely auxiliary quantities and have no rigorous physical meaning. However, this caveat is often ignored in practice. ${ }^{10}$ Now, let us compare our method with other, more approximate KSO approaches. It is quite straightforward to obtain approximate state-resolved cross sections directly from the time-dependent KSO. This is similar in spirit to the calculation of the ion probabilities from the KSO that can be found in Ref. 14. All one needs to do is project the time-dependent KSO onto the eigen-state wave-functions of $\mathrm{H}(1 s), \mathrm{H}(2 s)$, and $\mathrm{H}(2 p)$ centered at proton.

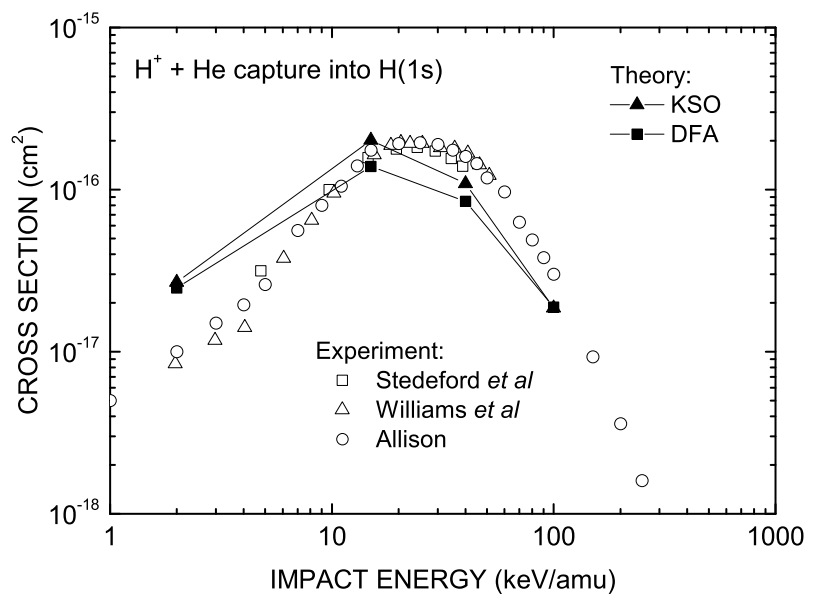

FIG. 6. Cross sections for electron capture into $\mathrm{H}(2 s)$ in $\mathrm{H}^{+}$on $\mathrm{He}\left(1 s^{2}\right)$. Experiment: $\triangleleft$, Ryding et al. $;^{42} \nabla$, Jaecks et al. $;^{43} \triangleright$, Hippler et al. $;^{44} \square$, Hughes et al. $;{ }^{37} \diamond$, Andreev et al. $;{ }^{34} \circ$, Crandall et al. $;{ }^{45} \Delta$, Fitzwilson et al. ${ }^{46}$ Theory: $\mathbf{m}$, present data; $\mathbf{\Lambda}$, from KSO method.

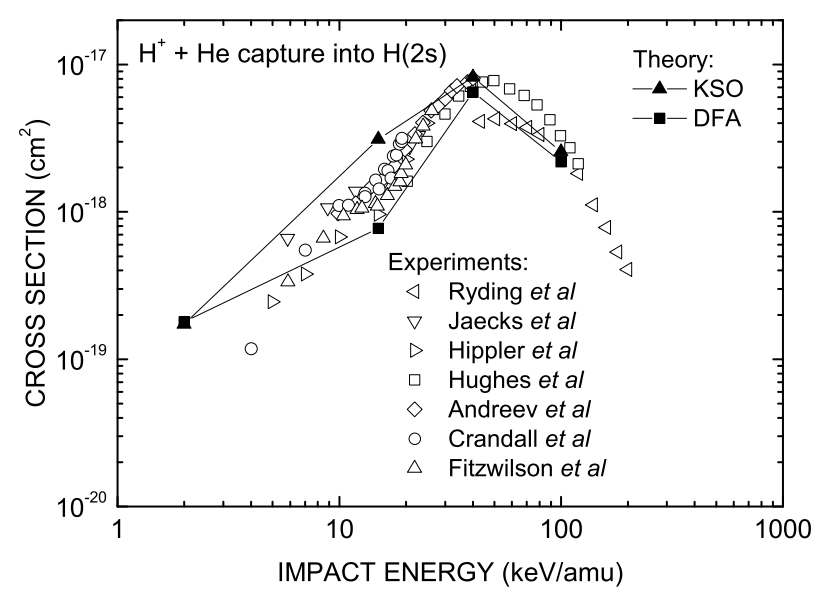

FIG. 7. Cross sections for electron capture into $\mathrm{H}(1 s)$ in $\mathrm{H}^{+}$on $\mathrm{He}\left(1 s^{2}\right)$. Experiment: $\square$, Stedeford et al. $;{ }^{47} \Delta$, Williams et al. $;^{48}$ O, Allison. ${ }^{49}$ Theory: $\mathbf{\square}$, present data; $\boldsymbol{\Lambda}$, from KSO method.

For the case of $\mathrm{H}^{+}+\mathrm{He}\left(1 s^{2}\right)$ scattering which initially has two electrons both in the $1 s$ orbital of the helium atom, the state-resolved probability to capture a single electron by proton is evaluated as

$$
P_{\chi}^{1}=\left(2-N_{a}^{\mathrm{H}}(t)\right)\left|\left\langle\phi_{H(\chi)} \mid \psi_{1 s}(t)\right\rangle\right|^{2},
$$

where $\phi_{\chi}$ is the eigen-state wave-function of the $\mathrm{H}(\chi)$ state centered at proton, $\psi_{1 s}(t)$ denotes the time-dependent KSO. Here and in what follows, we would like to refer the algorithm that presented Equation (9) as the KSO method.

Figures 6 and 7 show the cross sections for electron capture into $\mathrm{H}(1 s)$ and $\mathrm{H}(2 s)$, respectively, calculated by the DFA and KSO methods. The two results are in reasonable agreement with the experiment measurement, as should be expected, giving a good check on the overall numerical methods. It is very interesting to see that the two results are more in agreement with each other in the high-energy regime as well as in the lower-energy regime, while the KSO results are totally larger than the DFA results in the medium-energy regime. In the medium-energy regime, DFA results decrease more rapidly than the KSO results as the impact energy decreases, and DFA results get closer to the experiment.

Finally, one should realize that the predictive power of TDDFT is subject to the accuracy of the approximations made in two essential ingredients, namely the timedependent XC potential and the functional for the physical observable, excluding numerical approximations that are normally controllable. It has been demonstrated that the timedependent $\mathrm{XC}$ potential discontinuity in a real-time simulation dramatically affects the population of the resulting spatially confined regions or isolated subsystems. This indicates the importance of a proper account of the discontinuities in TDDFT descriptions of ionization, dissociation, or charge transfer processes. ${ }^{50}$ In the present study, our benchmark calculations rely on the adiabatic local-density approximation (ALDA) for the time-dependent XC potential, which is local in time, i.e., has no memory effects, and has no account of the discontinuities. However, one should realize that whether an approximation appears accurate or not depends on what 
is being measured and how sensitive the observable is, and on the details of the external perturbation. Again, we would also like to stress that the benchmark problem selected here is a very severe test of DFA algorithm, since one deals here with an extremely strongly correlated, strongly perturbed few-electron system. In view of this, of particular interest to us is that, the use of $L\left(N_{a}\right)$ seems a breakthrough in the handling of DFA algorithm, making it possible to use a very simple ALDA for the time-dependent XC potential in practical applications of TDDFT to treat systems with a fractional number of electrons, thus it deserves further development.

\section{CONCLUSIONS}

In summary, we have derived and carried out a computational method to extract the state-resolved probability from the knowledge of only the time-dependent density, in the spirit of TDDFT, taking as an example the $\mathrm{H}^{+}+\mathrm{He}\left(1 s^{2}\right)$ colliding system. Very good overall agreement between experimental and theoretical data is found, which provides further evidence for the applicability of the DFA algorithm to rather complex many-electron systems. Of course there were lots of other avenues to be explored, one conclusion that can be drawn at this stage is that the DFA algorithm demonstrates its high promise as a new tool in the arsenal of TDDFT methods.

\section{ACKNOWLEDGMENTS}

This work is supported in part by NSFC (Grant Nos. 10876003 and 11474020).

${ }^{1}$ M. A. L. Marques, C. A. Ullrich, F. Nogueira, A. Rubio, K. Burke, and E. K. U. Gross, Time-Dependent Density Functional Theory (Springer-Verlag, Heidelberg, 2006).

${ }^{2}$ M. A. L. Marques, N. T. Maitra, F. Nogueira, E. K. U. Gross, and A. Rubio, Fundamentals of Time-Dependent Density Functional Theory (SpringerVerlag, Heidelberg, 2012).

${ }^{3}$ C. A. Ullrich, Time-Dependent Density-Functional Theory: Concepts and Applications (Oxford University Press, 2012).

${ }^{4} \mathrm{~K}$. Burke et al., "Time-dependent density functional theory: Past, present, and future," J. Chem. Phys. 123, 062206 (2005).

${ }^{5}$ E. Runge et al., "Density-functional theory for time-dependent systems," Phys. Rev. Lett. 52, 997 (1984).

${ }^{6} \mathrm{M}$. Mundt et al., "Derivative discontinuities in time-dependent densityfunctional theory," Phys. Rev. Lett. 95, 203004 (2005).

${ }^{7}$ F. Calvayrac et al., "Nonlinear electron dynamics in metal clusters," Phys. Rep. 337, 493 (2000).

${ }^{8} \mathrm{~F}$. Wilken et al., "Adiabatic approximation of the correlation function in the density-functional treatment of ionization processes," Phys. Rev. Lett. 97, 203001 (2006).

${ }^{9} \mathrm{~N}$. Rohringer et al., "Calculating state-to-state transition probabilities within time-dependent density-functional theory,” Phys. Rev. A 74, 042512 (2006).

${ }^{10}$ Y. H. Li et al., "Time-dependent transition density matrix," Chem. Phys. 391, 157 (2011).

${ }^{11} \mathrm{~F}$. Wang et al., "An effective method for state population within timedependent density functional theory," J. Chem. Phys. 139, 094108 (2013).

${ }^{12}$ P. O. Löwdin, "Quantum theory of many-particle systems. I. Physical interpretations by means of density matrices, natural spin-orbitals, and convergence problems in the method of configurational interaction," Phys. Rev. 97, 1474 (1955).
${ }^{13}$ J. P. Perdew et al., "Density-functional theory for fractional particle number: Derivative discontinuities of the energy," Phys. Rev. Lett. 49, 1691 (1982).

${ }^{14}$ C. A. Ullrich, "Time-dependent Kohn-Sham approach to multiple ionization," J. Mol. Struct.: THEOCHEM 501-502, 315 (2000).

${ }^{15}$ D. C. Montgomery and E. A. Peck, Introduction to Linear Regression Analysis, 2nd ed. (John Wiley and Sons, Inc., 1992).

${ }^{16} \mathrm{~J}$. B. Delos et al., "Semiclassical theory of inelastic collisions. II. Momentum-space formulation," Phys. Rev. A 6, 720 (1972).

${ }^{17} \mathrm{~A}$. Kołakowska et al., "Excitation and charge transfer in proton-hydrogen collisions," Phys. Rev. A 58, 2872 (1998).

${ }^{18} \mathrm{~A}$. Castro et al., "Propagators for the time-dependent Kohn-Sham equations," J. Chem. Phys. 121, 3425 (2004).

${ }^{19}$ J. G. Muga et al., "Complex absorbing potentials," Phys. Rep. 395, 357 (2004).

${ }^{20} \mathrm{Q}$. Chen et al., "Non-adiabatic time-dependent mean-field description for ion-atom collisions: Application to $\mathrm{H}^{+}+\mathrm{Na}(3 \mathrm{~s}) \mathrm{H}^{+}+\mathrm{Na}(3 \mathrm{p})$ case," J. Phys. B 38, 4291 (2005).

${ }^{21} \mathrm{~F}$. Wang et al., "Coordinate space translation technique for simulation of electronic process in the ion-atom collision," J. Chem. Phys. 134, 154308 (2011).

${ }^{22} \mathrm{~F}$. Wang et al., "A theoretical model for electron transfer in ion-atom collisions: Calculations for the collision of a proton with an argon atom," Phys. Lett. A 375, 3290 (2011).

${ }^{23} \mathrm{~F}$. Wang etal., "Comparison of three methods for calculation of electron transfer probability in $\mathrm{H}^{+}+\mathrm{Ne}$," Phys. Lett. A 376, 469 (2012).

${ }^{24} \mathrm{C}$. L. Zhang et al., "Theoretical investigation of $\mathrm{He}^{2+}-\mathrm{Ar}$ collisions in the energy range of 4-300 keV/amu," Phys. Rev. A 87, 032711 (2013).

${ }^{25} \mathrm{X}$. H. Hong et al., "Theoretical investigation of the electron capture and loss processes in the collisions of $\mathrm{He}^{2+}+\mathrm{Ne}$, J J. Chem. Phys. 139, 084321 (2013).

${ }^{26} \mathrm{X}$. H. Hong et al., " $\mathrm{H}^{+}-\mathrm{H}_{2} \mathrm{O}$ collisions studied by time-dependent densityfunctional theory combined with the molecular dynamics method," Phys. Rev. A 93, 062706 (2016).

${ }^{27} \mathrm{~N}$. Troullier et al., "Efficient pseudopotentials for plane-wave calculations," Phys. Rev. B 43, 1993 (1991).

${ }^{28} \mathrm{M}$. Oliveira et al., "Generating relativistic pseudo-potentials with explicit incorporation of semi-core states using APE, the Atomic Pseudo-potentials Engine," Comput. Phys. Comm. 178, 524 (2008).

${ }^{29}$ M. A. L. Marques et al., "octopus: A first-principles tool for excited electronion dynamics," Comput. Phys. Commun. 151, 60 (2003).

${ }^{30}$ A. Castro et al., "octopus: A tool for the application of time-dependent density functional theory," Phys. Status Solidi B 243, 2465 (2006).

${ }^{31}$ D. M. Ceperley et al., "Ground state of the electron gas by a stochastic method," Phys. Rev. Lett. 45, 566 (1980).

${ }^{32}$ J. P. Perdew et al., "Self-interaction correction to density-functional approximations for many-electron systems," Phys. Rev. B 23, 5048 (1981).

${ }^{33}$ R. Hippler et al., "Cross sections for charge-exchange excitation to $\mathrm{H}(2 \mathrm{p})$ in proton-rare-gas-atom collisions (1-25 keV)," Phys. Rev. A 35, 3139 (1987).

${ }^{34}$ E. P. Andreev et al., "Charge exchange of protons in inert gases involving the formation of fast hydrogen atoms in the $2 \mathrm{~s}$ and $2 \mathrm{p}$ states," Sov. Phys. JETP 23, 375 (1966)

${ }^{35}$ T. D. Gaily et al., "Polarization of Lyman-alpha radiation from $\mathrm{H}^{+}-$and $\mathrm{D}^{+}-$ rare-gas charge-transfer collisions," Phys. Rev. 167, 81 (1968).

${ }^{36} \mathrm{R}$. Hippler et al., "Alignment of $\mathrm{H}(2 \mathrm{p})$ following $\mathrm{H}^{+}-\mathrm{He}$, Ar chargechanging collisions," J. Phys. B 19, 1507 (1986).

${ }^{37} \mathrm{R}$. H. Hughes et al., "Electron capture into the $\mathrm{n}=2$ states of hydrogen by fast proton impact on gases," Phys. Rev. A 4, 1453 (1971).

${ }^{38}$ D. Pretzer et al., "Lyman alpha production in proton-rare gas collisions," Phys. Rev. Lett. 10, 340 (1963).

${ }^{39} \mathrm{~B}$. Van Zyl et al., "Lyman- emission from $\mathrm{H}^{+}$impact on rare-gas atoms," Phys. Rev. A 35, 4551 (1987).

${ }^{40} \mathrm{~J}$. S. Risley et al., "Electron-transfer cross sections to low-lying excited states of hydrogen in collisions of protons with helium and argon,” J. Phys. B 11, 1759 (1978).

${ }^{41}$ R. K. Janev, Atomic and Plasma-Material Interaction Data for Fusion (Supplement to the Journal Nuclear Fusion) (International Atomic Energy Agency, Vienna, 1992), Vol. 3, p. 63.

${ }^{42}$ S. Ryding et al., "A study of Lyman emission in charge-transfer collisions involving 40-200 keV protons," Proc. Phys. Soc. 89, 547 (1966).

${ }^{43}$ D. H. Jaecks et al., "Production of metastable hydrogen atoms in protonrare-gas collisions," Phys. Rev. 137, A340 (1965). 
${ }^{44}$ R. Hippler et al., "Coherent excitation of $\mathrm{H}(\mathrm{n}=2)$ in $\mathrm{H}^{+}, \mathrm{H}-\mathrm{He}$ collisions," Z. Phys. D: At., Mol. Clusters 18, 61 (1991).

${ }^{45}$ D. H. Crandall et al., "Charge transfer to the 2 s state of hydrogen from close collisions of $\mathrm{H}^{+}$with He and Ar," Phys. Rev. A 4, 2271 (1971).

${ }^{46}$ R. L. Fitzwilson et al., "Formation of metastable hydrogen atoms by charge transfer," Phys. Rev. A 3, 1305 (1971).

${ }^{47}$ J. B. H. Stedeford et al., "Further investigations of charge exchange and electron detachment. I. Ion energies 3 to $40 \mathrm{keV}$. II. Ion energies 100 to 4000 eV," Proc. R. Soc. A 227, 466 (1955).
${ }^{48}$ J. F. Williams et al., "Charge exchange and dissociation cross sections for $\mathrm{H}_{1}^{+}, \mathrm{H}_{2}^{+}$, and $\mathrm{H}_{3}^{+}$ions of 2- to 50-kev energy incident upon hydrogen and the inert gases," Phys. Rev. 149, 62 (1966).

${ }^{49}$ S. K. Allison, "Experimental results on charge-changing collisions of hydrogen and helium atoms and ions at kinetic energies above $0.2 \mathrm{kev}$," Rev. Mod. Phys. 30, 1137 (1958).

${ }^{50} \mathrm{D}$. Vieira et al., "Physical signatures of discontinuities of the time-dependent exchange-correlation potential," Phys. Chem. Chem. Phys. 11, 4647 (2009). 